\title{
Change: The Future of Pharmacy
}

\author{
Patricia Macgregor
}

$\mathrm{I}$ n August 2015, the Canadian Society of Hospital Pharmacists (CSHP) will launch a new strategic plan to guide the Society in meeting the needs of pharmacists from hospitals and other collaborative health care settings over the next few years. The CSHP 2015 project will be complete, and we hope that its legacy of promoting clinical excellence continues to grow, that the culture of knowledge translation and the sharing of successes live on, and that the commitment to and enhancements of patient care and outcomes remain.

The pharmacy profession deals with ongoing major challenges, from procurement to bedside. The management of drug shortages has prompted increased collaboration across pharmaceutical sectors in the prevention of future shortages. The scope of practice for both pharmacists and pharmacy technicians has expanded to varying degrees across the country, with pharmacist roles now including prescribing, injection, health promotion, and prevention and treatment of disease. Changes have been introduced to regulate compounding practices and hospital pharmacy accreditation. Requirements for accountability and outcome measures have grown, intensifying the scrutiny of clinical pharmacy and prompting in turn the development of validated performance indicators.

In addition, the complexity of patient care escalates, further increasing demands on pharmacists, but also providing opportunities for knowledge development and specialization in pharmacy practice. In particular, hospital practice models are changing toward more clinical and integrated models of care, and discussions are arising about the need and desire for specialty certification in Canada.

The transition to the PharmD as the entry-to-practice degree for pharmacists and a corresponding increase in the need for student placements are affecting hospital training programs and demands on pharmacists, challenging current infrastructure and approaches. New models of experiential education in hospitals and primary care are required to meet these growing needs.

The common factor in all this is change: in education and training, in clinical practice, in work processes, in professional scope and expertise, in regulatory standards. Embracing change is essential to managing it. Infrastructure and resources are necessary enablers to implementing change, and measurement and reporting are important tools for communicating the impact of change.

CSHP 2015 has

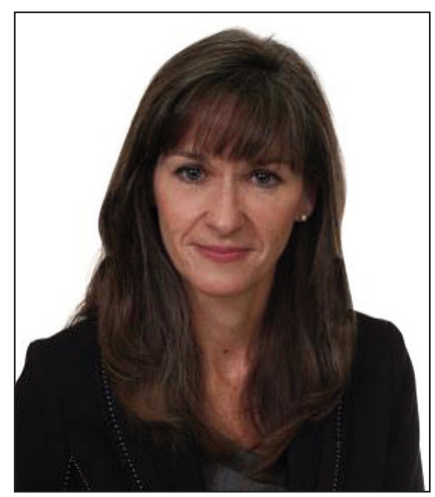

provided a framework for practice excellence and measurement of progress. The Canadian National Clinical Pharmacy Key Performance Indicators Collaborative, in partnership with CSHP, will soon be providing knowledge translation for measurement and reporting of outcomes. The Association of Faculties of Pharmacy of Canada, partially supported by the Blueprint for Pharmacy, is creating a preceptor development program and models of experiential education to facilitate the growing demand for student training.

Through these times of change and opportunity, CSHP will continue to support its members by seeking answers to the issues that challenge us: What will pharmacy look like in the future? What will be the needs of pharmacists in hospitals and collaborative health care settings, in terms of education, guidelines, specialization, and professional recognition? How will pharmacy practice be structured and regulated? These and other questions will inform the new CSHP strategic plan and will be the focus of activities in the years to come.

Patricia Macgregor, BSc, RPh, MRPharmS, MHSc, CHE, is Past President and Vision Liaison for the Canadian Society of Hospital Pharmacists. 